\title{
Improving the Cost Factor of DLBCA Lightweight Block Cipher Algorithm
}

\author{
Sufyan Salim Mahmood Al-Dabbagh*1, Alyaa Ghanim Sulaiman², Imad Fakhri Taha Al Shaikhli³, \\ Khalid Abdulkareem Al-Enezi ${ }^{4}$, Abdulrahman Yousef Alenezi ${ }^{5}$ \\ ${ }^{1}$ Department of Computer Science, University of Mosul, Iraq \\ ${ }^{2}$ Department of Software Engineering, University of Mosul, Iraq \\ ${ }^{3}$ Department of Computer Science, International Islamic University, Malaysia \\ ${ }^{4}$ Central Agency for Information Technology, Kuwait \\ ${ }^{5}$ Technology \& Infrastructure Department, Zain Kuwait Telecom Company, Kuwait
}

\begin{tabular}{|c|c|}
\hline Article Info & ABSTRACT \\
\hline Article history: & ding to secure information in restricted environments is very \\
\hline Received Nov 19, 2017 & $\begin{array}{l}\text { important so that lightweight block cipher algorithm is suitable for these } \\
\text { environments. This paper improved DLBCA algorithm by decreasing the }\end{array}$ \\
\hline Revised Jan 21, 2018 & cost factor through using the less number of S-boxes. Also, differential and \\
\hline Accepted Feb 24, 2018 & $\begin{array}{l}\text { boomerang attacks have been applied in this paper. Finally, all the results } \\
\text { have been presented. }\end{array}$ \\
\hline
\end{tabular}

Keywords:

Cost factor

Cryptanalysis

Lightweight algorithms

Copyright $(2018$ Institute of Advanced Engineering and Science. All rights reserved.

\section{Corresponding Author:}

Sufyan Salim Mahmood Al-Dabbagh

Computer Science Dept.,

College of computer science and mathematics,

Mosul University, Mosul, Iraq.

Email: sufyansalim_77@yahoo.com

\section{INTRODUCTION}

Information technology is changing tremendously, and the security system is needed to protect data [1]. Generally, it is not easy to suggest a cryptographic algorithm for all kinds of target devices [2]. There are three factors cost, security and performance. The designer must be aware how to use these factors [2]. There are many proposed lightweight algorithms like TWINE [9], PRINT [4], KLEIN [7], PRESENT [5], LBLOCK [8], mCrypton [6], PRINCE [3] and LED [10].

This research paper, will enhance the cost element of DLBCA algorithm by reducing the number of S-box without major change on other elements. Also, two attacks differential and boomerang have been applied on the suggested algorithm.

\section{DLBCA LIGHTWIGH ALGORITHM}

DLBCA is 32-bit plaintext and key size 80-bit. The structure of DLBCA algorithm looks like the structure of feistel with some modifications [11]. There are 32 rounds and in each round, there are operations like: Substitution box, Bit permutation, XOR, Rotation and key update. Moreover, there is XOR between the cipher text and key in the last round. The DLBCA have four layers as following: 
- First Layer: in this layer, the 32-bit plaintext is XOR with the 32-bit key. The plaintext divides into two parts. Each part is 16-bit and the results after XOR of left part will be as inputs to the second layer (Substitution box).

- Second Layer: this layer is the most important layer. It produces the confusion property and it gives the nonlinearity to the algorithm. It has four 4-bit S-boxes. The output of this layer will be as inputs to the third layer (bit permutation). Also, this layer uses one S-box and repeats it 8 times. The characteristics of the S-box are the same with good S-box. The values of S-box as shown in Table (1).

Table 1 S-Box Values

\begin{tabular}{cccccccccccccccccc}
\hline $\mathrm{X}$ & 0 & 1 & 2 & 3 & 4 & 5 & 6 & 7 & 8 & 9 & $\mathrm{~A}$ & $\mathrm{~B}$ & $\mathrm{C}$ & $\mathrm{D}$ & $\mathrm{E}$ & $\mathrm{F}$ \\
\hline $\mathrm{S}(\mathrm{X})$ & $\mathrm{F}$ & $\mathrm{C}$ & 2 & 7 & 9 & 0 & 5 & $\mathrm{~A}$ & 1 & $\mathrm{~B}$ & $\mathrm{E}$ & 8 & 6 & $\mathrm{D}$ & 3 & 4 \\
\hline
\end{tabular}

- Third Layer: This layer produces the diffusion which is also important part for any strong encryption algorithm. This method of bit permutation applies on left side which is 16-bit.

- Fourth Layer: this layer applies the rotation and XOR operations on both sides. First of all, rotate the left 16-bit and then XOR with right 16-bit. The result will keep in left 16-bit. The next step is to rotate the right 16-bit and XOR with new left 16-bit and the result will keep in right 16-bit.

The last important part in any encryption algorithm is key schedule. The MASTER key size as mentioned before is 80 -bit $\mathrm{K} 0, \mathrm{~K} 1, \mathrm{~K} 2, \mathrm{~K} 3, \mathrm{~K} 4, \ldots . \mathrm{K} 79$. The key update or key schedule is operate as in [17].

\section{PROPOSED LIGHTWEIGHT ALGORITHM}

This paper enhances the cost factor of DLBCA by reducing the S-box numbers. The suggested algorithm utilizes four S-box instead of eight S-box. However, it has xoring 32bit of plaintext with first 32bit of key. The round number of suggested algorithm is similar as DLBCA. The suggested algorithm layers are shown in Figure (2).

\section{THE DISCUSSION OF COST}

The cost element is one of essential factors that the designer must be consider it when he designs any algorithm. This paper calculated the cost of suggested algorithm according to [12]. The cost calculating details as follows:

a. The saving value of 1 bits is $6 \mathrm{GE}$ while cost value of one $\mathrm{S}$-box is $22 \mathrm{GE}$.

b. The cost of 16-bit XOR is 43.5GE.

c. For additional cost, there is 50GE.

The costs of the algorithms are presented in table (2).

Table 2: Cost Comparison between Proposed Algorithm and Others Algorithms

\begin{tabular}{ccccc}
\hline Algorithm & Plaintext & Key & S-box & Cost \\
\hline Lblock [9] & 64 & 80 & 8 & $1320 \mathrm{GE}$ \\
TWINE [10] & 64 & 80 & 8 & $1503 \mathrm{GE}$ \\
PRESENT [6] & 64 & 80 & 16 & $1570 \mathrm{GE}$ \\
KLIEN [8] & 64 & 80 & 16 & $2097 \mathrm{GE}$ \\
DLBCA [ 19] & 32 & 80 & 8 & $1116 \mathrm{GE}$ \\
Proposed algorithm & 32 & 80 & 4 & $1028 \mathrm{GE}$ \\
\hline
\end{tabular}

The cost of the suggested algorithm is the lowest comparing with the other algorithms as shown in table (2) 


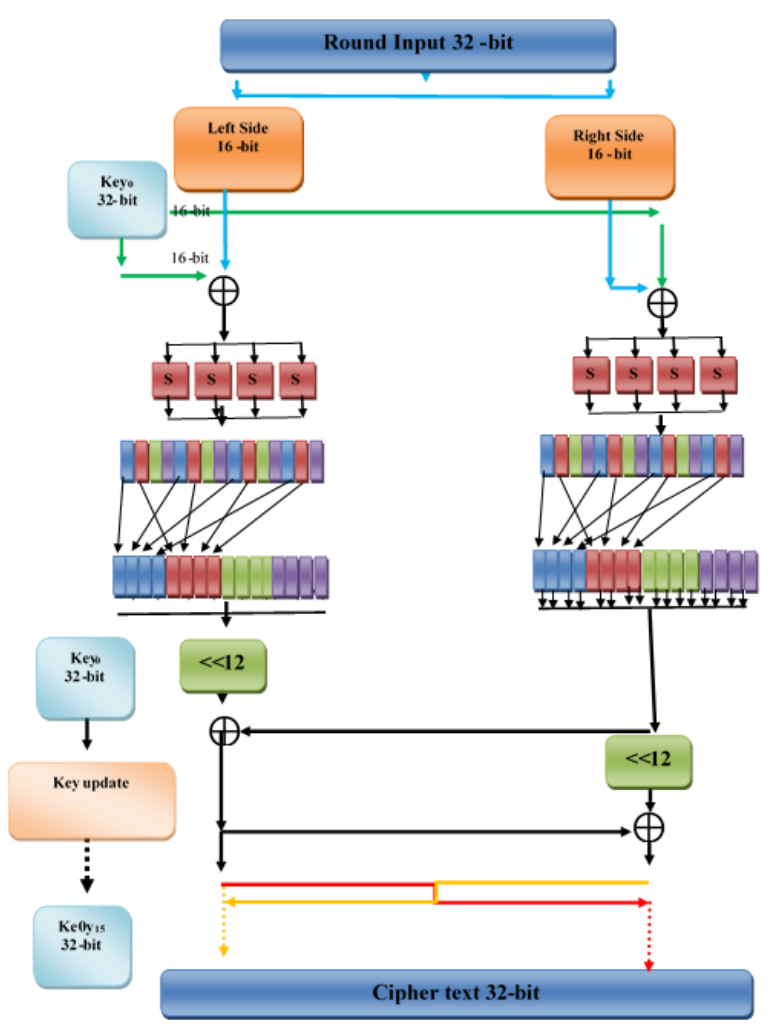

Figure 1. DLBCA Algorithm Layers in Details [17]

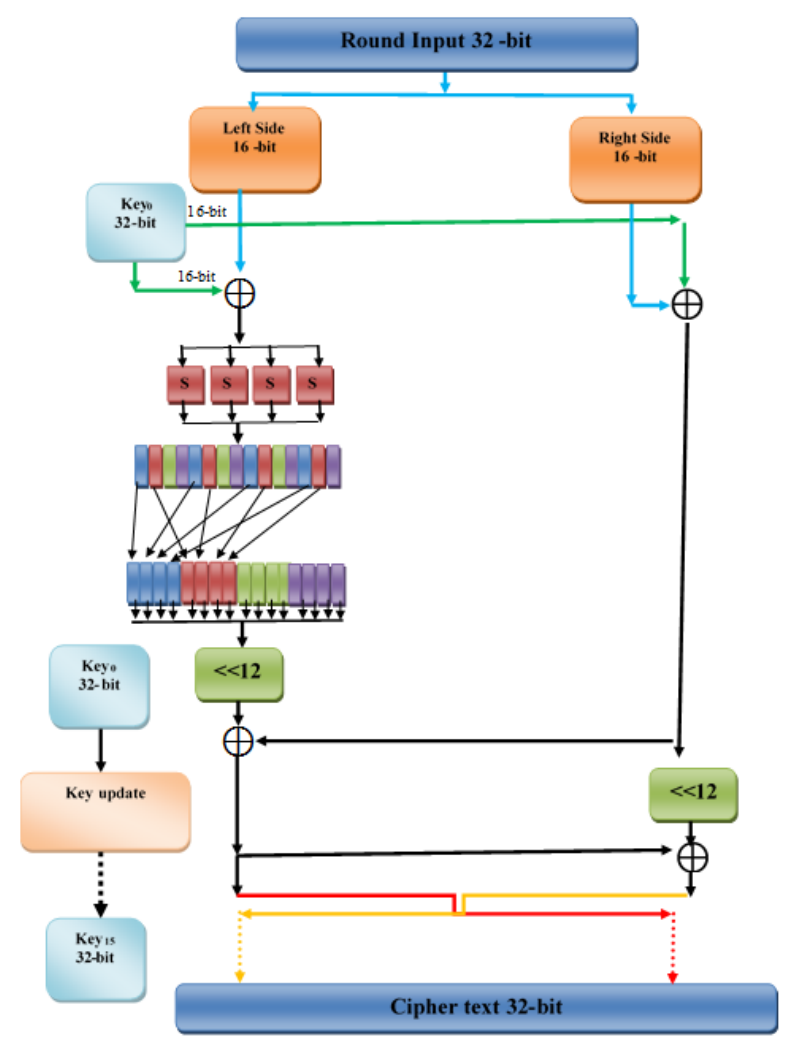

Figure 2. Suggested Algorithm Layers in Details 


\section{THE DISCUSSION OF SECURITY}

The cryptanalysis is essential tool that it can scale the security of whatever algorithm. Differential and boomerang attacks are applied in this paper.

\subsection{Differential Cryptanalysis}

The minimum active S-box is the most powerful way to measure the resistance of block cipher algorithm against differential algorithm [13]-[15]. The results of this attack are presented in table (3).

Table 3. Active S-box Numbers for Suggested Algorithm and Other Algorithms

\begin{tabular}{clcccc}
\hline No. & Algorithm & \multicolumn{4}{c}{ Min number of active S-box } \\
& & 4 & 8 & 12 & 16 \\
\hline 1. & TWINE [10] & 3 & 11 & 24 & - \\
2. & Lblock [9] & 3 & 11 & 24 & 35 \\
3. & PRESENT [6] & 8 & 16 & 24 & 32 \\
4. & KLEIN [8] & 15 & 30 & 45 & 60 \\
5. & DLBCA [17] & 13 & 31 & 48 & 65 \\
6. & Proposed algorithm & 8 & 16 & 24 & 32 \\
\hline
\end{tabular}

The Table (3) shows that the number of active S-boxes of LBLOCK, PRESENT and TWINE is closer to suggested algorithm while the number of active S-box of DLBCA and KLEIN are better than our suggested algorithm. On the other hand, the cost of both of them are higher compared with this suggested algorithm.

\subsection{Boomerang Cryptanalysis}

Knowing the active S-box numbers in each step is the first step to amount this attack while the second step is calculating the distinguisher probability for this attack. The Equation is:

$$
p^{2} \cdot q^{2}=\left(\left(\left(2^{-2}\right)^{N A S}\right)^{2} \times\left(\left(2^{-2}\right)^{N A S}\right)\right)^{2}
$$

Which $\mathrm{p}^{\wedge} 2 . \mathrm{q}^{\wedge} 2$ is the distinguisher probability and NAS is the active S-box. When the probability of distinguisher is less than $2^{- \text {plaintext size }}$, we can say the attack can't go forward [16].

Regarding to the proposed algorithm and depending on equation (1), this attack can reach round 3 with maximal probability $2^{-28}$. The following points will explain that:

a. In round 2 there are 4 active $S$-boxes and in round 1 there is two active S-box.

b. To find the probability, we need to apply the equation (1).

- The final probability is $\left(\left(\left(2^{-2}\right)^{4}\right)^{2}\right) \times\left(\left(\left(2^{-2}\right)^{2}\right)^{2}\right)=2^{-16} \times 2^{-8}=2^{-24}$.

c. This attack can reach 3 rounds only with probability $2^{\wedge}(-24)$.

The proposed algorithm has 32 rounds meaning that it is resistant to the boomerang attack. The results of this attack are presented in table (4).

\begin{tabular}{|c|c|c|c|}
\hline & No. & Algorithm & $\begin{array}{l}\text { Maximum } \\
\text { Round }\end{array}$ \\
\hline & 1 & LBLOCK [9] & 11 \\
\hline & 2 & TWINE [5] & 11 \\
\hline & 3 & PRESENT [6] & 7 \\
\hline & 4 & KLEIN [8] & 4 \\
\hline & 5 & DLBCA [17] & 3 \\
\hline & 6 & Proposed & 3 \\
\hline
\end{tabular}

The Table (4) shows that boomerang cryptanalysis can reach to round 11 with TWINE and LBLOCK algorithms but it can reach to round 7 and round 4 with PRESENT and KLEIN respectively.

While the boomerang cryptanalysis can reach round 3 with DLBCA and suggested algorithm. This is mean that the suggested algorithm still has the same number of rounds which is equal with DLBCA after reducing the cost factor. Also, the suggested algorithm is better than other algorithms regarding of boomerang cryptanalysis. 


\section{CONCLUSION}

This paper has improved the cost factor of DLBCA by reducing the number of S-box. Also, the comparisons for cost and security between suggested algorithm and others are shown. To sum up results, the cost of suggested algorithm is the lowest cost. Regarding to security side, the suggested algorithm has similar number of active S-box when compared with algorithms (TWINE, LBLOCK and PRESENT). According to the boomerang attack, the suggested algorithm has the same number of rounds with DLBCA algorithm and it is the best from other algorithms.

\section{REFERENCES}

[1] Panasenko, S., \& Smagin, S., "Lightweight Cryptography: Underlying Principles and Approaches", International Journal of Computer Theory and Engineering, Vol 3 No.4, (2011).

[2] S. Salim and I. Taha, "Lightweight block ciphers: comparative study," Journal of Advanced Computer Science and Technology Research (JACSTR), vol. 2, pp. 159-165, 2012.

[3] J. Borghoff, et al., "PRINCE - A Low-Latency Block Cipher for Pervasive Computing Applications," in Advances in Cryptology - ASIACRYPT 2012. vol. 7658, Springer Berlin Heidelberg, 2012, pp. 208-225.

[4] L. Knudsen, et al., "PRINTcipher: A Block Cipher for IC-Printing," in Cryptographic Hardware and Embedded Systems, CHES 2010. vol. 6225, Springer Berlin Heidelberg, 2010, pp. 16-32.

[5] A. Bogdanov, L. Knudsen, G. Leander, C. Paar, A. Poschmann, M. Robshaw, Y. Seurin, and C. Vikkelsoe, "PRESENT: An Ultra-Lightweight Block Cipher Cryptographic Hardware and Embedded Systems - CHES 2007." Vol. 4727, Springer Berlin / Heidelberg, 2007, pp. 450-466.

[6] C. Lim and T. Korkishko, "mCrypton - A Lightweight Block Cipher for Security of Low-Cost RFID Tags and Sensors Information Security Applications." Vol. 3786, Springer Berlin / Heidelberg, 2006, pp. 243-258.

[7] Z. Gong, S. Nikova, and Y. Law, "KLEIN: A New Family of Lightweight Block Ciphers RFID. Security and Privacy." Vol. 7055, Springer Berlin / Heidelberg, 2012, pp. 1-18.

[8] W. Wu and L. Zhang, "LBlock: A Lightweight Block Cipher Applied Cryptography and Network Security." Vol. 6715, Springer Berlin / Heidelberg, 2011, pp. 327-344

[9] T. Suzaki, et al., "TWINE: A Lightweight Block Cipher for Multiple Platforms," in Selected Areas in Cryptography. vol. 7707, Springer Berlin Heidelberg, 2013, pp. 339-354.

[10] J. Guo, T. Peyrin, A. Poschmann, and M. Robshaw, "The LED Block Cipher Cryptographic Hardware and Embedded Systems - CHES 2011." Vol. 6917, Springer Berlin / Heidelberg, 2011, pp. 326-341.

[11] S. S. M. Aldabbagh and I. F. T. A. Shaikhli, "OLBCA: A New Lightweight Block Cipher Algorithm," in Advanced Computer Science Applications and Technologies (ACSAT), 2014 3rd International Conference on, 2014, pp. 1520.

[12] S. Panasenko and S. Smagin, "Lightweight cryptography: Underlying principles and approaches," International Journal of Computer Theory and Engineering, vol. 3, pp. 516-520, 2011.

[13] E. Biham and A. Shamir, "Differential Cryptanalysis of DES Variants," in Differential Cryptanalysis of the Data Encryption Standard, ed: Springer, 1993, pp. 33-77.

[14] J.-S. Kang, et al., "Practical and provable security against differential and linear cryptanalysis for substitutionpermutation networks," ETRI journal, vol. 23, pp. 158-167, 2001.

[15] E. Biham and A. Shamir, "Differential cryptanalysis of DES-like cryptosystems," Journal of CRYPTOLOGY, vol. 4, pp. 3-72, 1991.

[16] D. Wagner, "The boomerang attack," in Fast Software Encryption, 1999, pp. 156-170.

[17] S. S. M. Aldabbagh, " Design 32-bit Lightweight Block Cipher Algorithm (DLBCA)" International Journal of Computer Applications vol. 166, pp. 17-20, 2017. 


\section{BIOGRAPHIES OF AUTHORS}

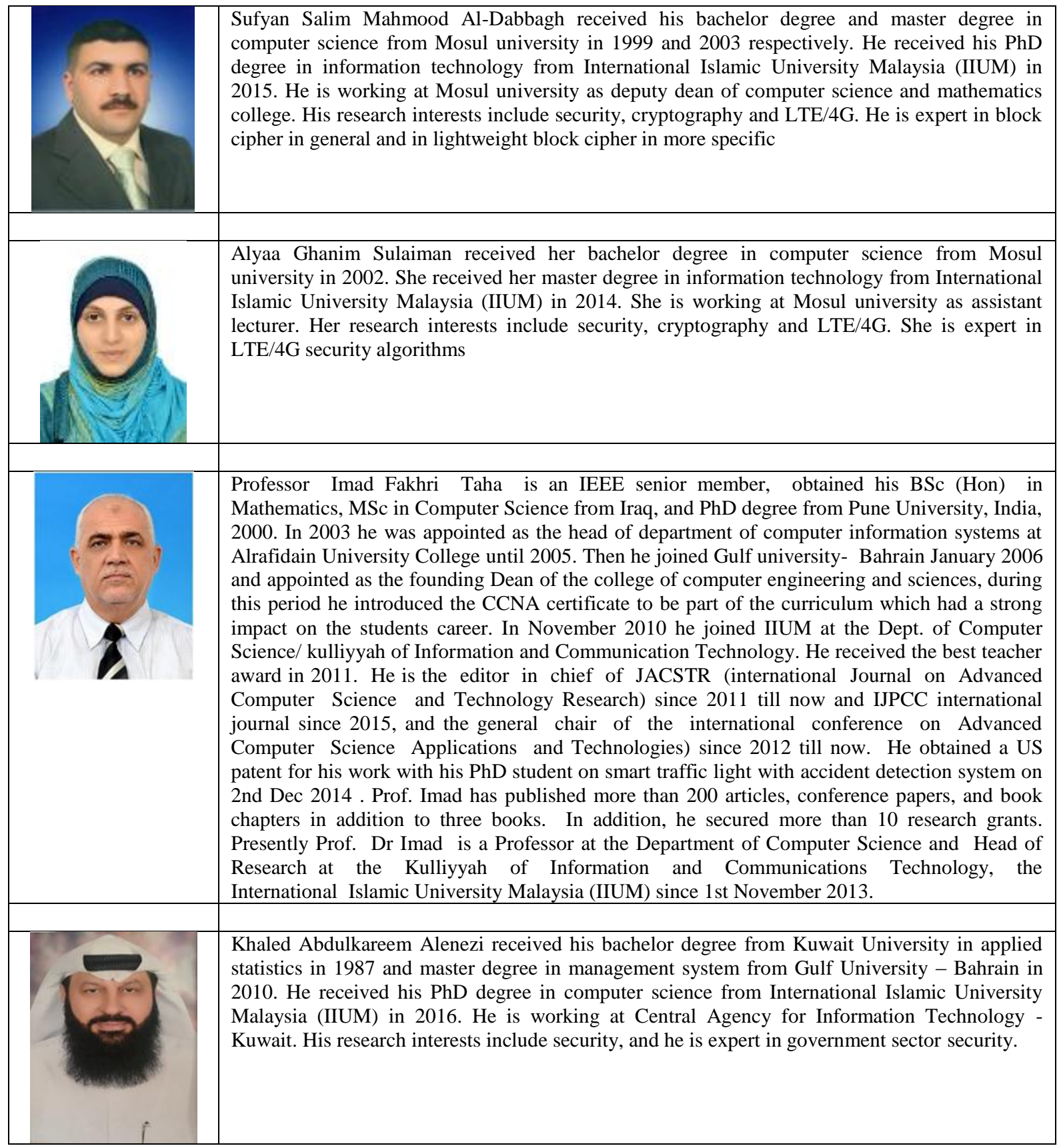

